\title{
The Application of Enoxaparin Sodium Injection in the Treatment of Cerebral Infarction
}

N. WANG* AND X. LIU

9th division, The 5th People's Hospital of Ji'nan, 24297 Jingshi Road, Huaiyin District, Jinan, 250022, Shandong Province, China

Wang and Liu: Healing Effect of PRF for Soft Tissue Defects in Oral Implants

The clinical efficacy of enoxaparin sodium injection on cerebral infarction was explored and the mechanism of action of enoxaparin sodium was analysed, which provided certain basis for the clinical antithrombotic treatment of acute cerebral infarction. A total of 68 patients with acute cerebral infarction treated in the 5th People's Hospital of Ji'nan from January 2016 to January 2019 were selected as the research subjects and were divided into the observation group and the control group through the random number table method, with 34 patients in each group. After being admitted to the hospital, patients in both groups were treated with routine treatments and nursing interventions by the Neurology Department; in addition, patients in the control group were given oral aspirin, and patients in the observation group were given oral aspirin and enoxaparin sodium injection. After being treated for 14 days, the clinical efficacy, European Stroke Scale results, and National Institutes of Health Stroke Scale results of patients in both groups were compared; in addition, the side effects of patients in both groups during the treatment courses 
were observed and recorded. The research results showed that the overall treatment effective rate in the observation group was $94.12 \%$, which was significantly $(p<0.05)$ higher than the $70.59 \%$ effective rate in the control group. The European Stroke Scale and National Institutes of Health Stroke Scale results of patients in both groups were improved after the treatment and the differences between the observation and the control group were statistically significant $(\mathbf{p}<\mathbf{0 . 0 5})$. In summary, the therapeutic effects of enoxaparin sodium injection in combination with aspirin were positive and such treatment plans could effectively improve the neurological deficiency without increasing the risks of haemorrhage, which was worth being widely applied clinically. However, the deficiencies of the research process, such as a small sample size and incomplete observation indicators, required improvement in subsequent research.

Key words: Enoxaparin sodium, cerebral infarction, low molecular weight heparin, anticoagulant, aspirin

Clinically, cerebral infarction is one of the most commonly seen cardiocerebrovascular diseases characterized by high disability rate, high mortality rate and high recurrence rate. Ischemic stroke is the symptom of neurological deficiency caused by the occlusion or stenosis of the cerebral vessels; its clinical features are sudden onset and rapid progression, leading to localized or diffuse brain function deficiencies ${ }^{[1]}$. The lesions of the cerebral arteries caused by atherosclerosis would result in stenosis or hardening of the vessels, or even develop into thrombosis, leading to the acute local interruption of cerebral perfusion, ischemia and hypoxia, softening and necrosis, and corresponding neurological symptoms, which would finally lead to hemiplegia, aphasia, coma, and death ${ }^{[2]}$.

In recent years, the incidence rate of cerebral infarction in China has increased annually. Therefore, the prevention and treatment of cerebral infarction are urgent. The antiplatelet drug has become the basic therapy for the prevention and treatment of atherosclerotic cerebral infarction, in which aspirin is one of the most effective antiplatelet drugs that have been certified by evidencebased medicine ${ }^{[3]}$. As a kind of classic anticoagulant, heparin has its unique advantages, such as strong anticoagulant effects and fast actions, and has been widely applied clinically. Compared with heparin, low molecular weight heparin has more uniform compositions and higher antiFXa/antiFlla ratio, whose antithrombotic activity is comparable to that of heparin; in addition, low molecular weight heparin also has high bioavailability, long half-life in vivo, low risks of hemorrhage, and can be administered by subcutaneous injections; besides, its pharmacokinetics features are predictable ${ }^{[4]}$. Therefore, low molecular weight heparin has been widely used in clinical practices, especially enoxaparin sodium.
Enoxaparin sodium is a patented drug of Aventis Corporation in France, which is produced by unfractionated heparin (HP) through alkaline $\beta$-elimination. The clinical indications of enoxaparin sodium include the prevention of acute deep vein thrombosis (DVT), the prevention of abdominal surgical and orthopedic operations, the treatment of patients with acute DVT accompanied by pulmonary embolism (PE), and the treatment of patients with non-PE-accompanied acute $\mathrm{DVT}^{[5,6]}$. In this clinical investigation, a total of 68 patients with cerebral infarction were selected as the subjects, based on routine neurological treatments and aspirin antiplatelet therapies, the therapeutic effects of enoxaparin sodium injections on cerebral infarction and the safety of the drug were observed to provide references and basis for the clinical treatments of cerebral infarction. The detailed research processes are demonstrated in the following sections.

A total of 68 patients with acute cerebral infarction treated in the 5th People's Hospital of Ji'nan from January 2016 to January 2019 were selected as the clinical subjects and were divided into the observation group and the control group using the random number table method, with 34 patients in each group. In the observation group, 21 cases were male and 13 cases were female, aged 43-79 y, with an average age of $62.30 \pm 5.46 \mathrm{y}$; once the patients suffered cerebral infarction, it took $4-18 \mathrm{~h}$ before receiving treatment with an average lag period of $13.17 \pm 1.31 \mathrm{~h}$. In the control group, 20 cases were male and 14 cases were female, aged 44-78 y, with an average age of $63.02 \pm 5.22 \mathrm{y}$; once the patients suffered cerebral infarction, it took $5 \sim 20 \mathrm{~h}$ before receiving treatment with an average lag period of $13.88 \pm 1.42 \mathrm{~h}$. All demographics of the patients in both groups, such as age, gender, and course of the disease, had no statistical significance $(\mathrm{p}<0.05)$, which is listed in Table 1 . The clinical study protocol was approved by 
TABLE 1: COMPARISONS OF GENERAL REFERENCES BETWEEN PATIENTS IN BOTH GROUPS

\begin{tabular}{|c|c|c|c|c|c|}
\hline \multirow{2}{*}{ Group } & \multirow{2}{*}{ Number } & \multicolumn{2}{|c|}{ Gender } & \multirow{2}{*}{ Age } & \multirow{2}{*}{ Onset time ( $\mathrm{h}$} \\
\hline & & Male & Female & & \\
\hline The observation group & 34 & $21(61.8 \%)$ & $13(38.2 \%)$ & $62.30 \pm 5.46$ & $13.17 \pm 1.31$ \\
\hline The control group & 34 & $20(58.8 \%)$ & $14(41.2 \%)$ & $63.02 \pm 5.22$ & $13.88 \pm 1.42$ \\
\hline$t / x^{2}$ & - & \multicolumn{2}{|c|}{11.232} & 7.554 & 6.249 \\
\hline$P$ value & - & \multicolumn{2}{|c|}{$<0.05$} & $<0.05$ & $<0.05$ \\
\hline
\end{tabular}

the Ethics Committee of the 5th People's Hospital of Ji'nan and all patients and their families had signed the informed consent forms.

Inclusion criteria were, patients who were positively diagnosed as acute cerebral infarction through computed tomography (CT) or magnetic resonance imaging; patients without obvious consciousness disorders; patients whose paralysis extremity myodynamia scores were $0 \sim 4$ grades; patients less than 80 y old; patients whose major manifestations were physical disability and lalopathy without consciousness disorders. Exclusion criteria were, patients who could not take the drugs used in this study; patients with active ulcers, active hemorrhage, or severe risks of hemorrhage; patients with severe cardiac dysfunction, atrial fibrillation, cavitary tuberculosis, or liver and renal dysfunctions.

After being admitted to the hospital, patients in both groups were treated with routine treatment and nursing intervention was given by the clinicians in the Neurology Department. The respiratory tracts were kept unobstructed, if necessary, oxygen support was given; nutritional support was provided to patients to maintain water and electrolyte balance and acid-base equilibrium; Protective treatments of brain functions were given and the intracranial pressure was reduced; blood pressures of patients with acute cerebral infarction should be maintained slightly higher than the normal level before the incidence of the disease; plasma glucose levels were controlled actively and complications such as the gastrointestinal hemorrhage, respiratory infections and pressure sores should be monitored.

Patients in the control group were given a single dose of $100 \mathrm{mg} / \mathrm{per}$ day aspirin orally in addition to the routine treatments for 14 consecutive days. In addition to the treatments given to the control group, patients in the observation group were given subcutaneously 4000 IU of enoxaparin sodium (Shenzhen Techdow Pharmaceutical Corporation, China; approval number: CFDA H20056850) twice daily for $14 \mathrm{~d}$.

The clinical efficacy of treatments given to patients in both groups was compared. Patients were subjected to on $\mathrm{d} 1,7$, and 14 after starting treatment, clinical neurological deficiency and the European Stroke Scale (ESS) assessments. The ESS evaluation standards were, (i) clinically cured, ESS $>96$ points, patients could work normally; (ii) clinically effective, ESS $>60$ points, patients could partially take care of themselves in daily lives and the disease conditions were improved; and (iii) invalid, ESS $<60$ points, no obvious improvement was seen after giving treatments ${ }^{[7]}$.

All patients were assessed by National Institutes of Health Stroke Scale (NIHSS) when they were admitted to the hospital and were assessed by NIHSS again after being treated for $14 \mathrm{~d}$; the comparison of therapeutic effects between groups was based on the variations of NIHSS scores ${ }^{[8]}$. The NIHSS score had 42 points in total; 1a (3 points $)+1$ b ( 2 points $)+1 \mathrm{c}(2$ points $)+2$ ( 2 points $)+3$ ( 3 points) +4 ( 3 points $)+5$ ( 4 points of the left upper $\operatorname{limb}+4$ points of the right upper limb) +6 (4 points of the left lower limb +4 points of the right lower limb) +7 ( 2 points $)+8$ ( 2 points $)+9$ ( 3 points $)+10$ ( 2 points) +11 (2 points) adding up to 42 points. However, since patients in coma or tetraplegia got 0 points for coordination exercise, the highest scores for these patients were only 40 points. The grades of points were that the higher the points were, the more severe the nerve damages were; the grades were as follows: (i) 0-1 point- normal or near normal; (ii) 1-4 points- mild to minor stroke; (iii) $5-15$ points- moderate stroke; (iv) $15-20$ points- moderate to severe stroke; and (v) 21-42 points- severe stroke ${ }^{[9]}$. The side effects of patients in both groups during the treatment were observed and recorded, including but not limited to erythra, hemorrhage and gastrointestinal discomfort.

SPSS 22.0 statistics software was applied to process the data obtained before and after treatments were given; the count data were expressed as $\mathrm{n} / \%$ and tested by $\chi^{2}$; the measurement data were expressed as mean \pm standard deviation and tested by independent sample t-test; $\mathrm{p}<0.05$ indicated statistical significance.

The results obtained suggested that the ESS results of patients in both groups before and $1 \mathrm{~d}$ after the treatments were not significantly different, while 7 and $14 \mathrm{~d}$ after the treatment, the ESS results of patients in both groups 
were improved compared to those obtained before the treatment. The improvement in the observation group was significantly greater than that in the control group $(p<0.05)$. The comparisons and variation tendencies of ESS results of patients in both groups were shown in Table 2.

In the acute stage of cerebral infarction, since the cerebral vessels were blocked, the cerebral perfusion was reduced, leading to insufficient blood supply to the brain, which would ultimately cause ischemia, hypoxic necrosis and neuronal damage. Aspirin is the current most widely applied antiplatelet drugs with proven therapeutic effects. Thromboxane A2 (TXA2) was not stored in the platelets, once the platelet was activated only then TXA2 is generated, as shown in fig. 1. TXA2 could initiate platelet aggregation. Aspirin irreversibly acetylates the cyclooxygenase- 1 of the platelets leading to reduced generation of TXA2.

The results of this investigation showed that after the treatment, the disease condition of patients in both groups was improved to different degrees compared to that before the treatment. In addition, the clinical effective rate of the observation group was significantly $(p<0.05)$ higher than that of the control group as shown in Table 3.

Before the treatment, differences in NIHSS results between patients in both groups were insignificant; but after the treatment, the NIHSS results of patients in both groups reduced, but significantly $(p<0.05)$ reduced in the observation group compared to that in the control

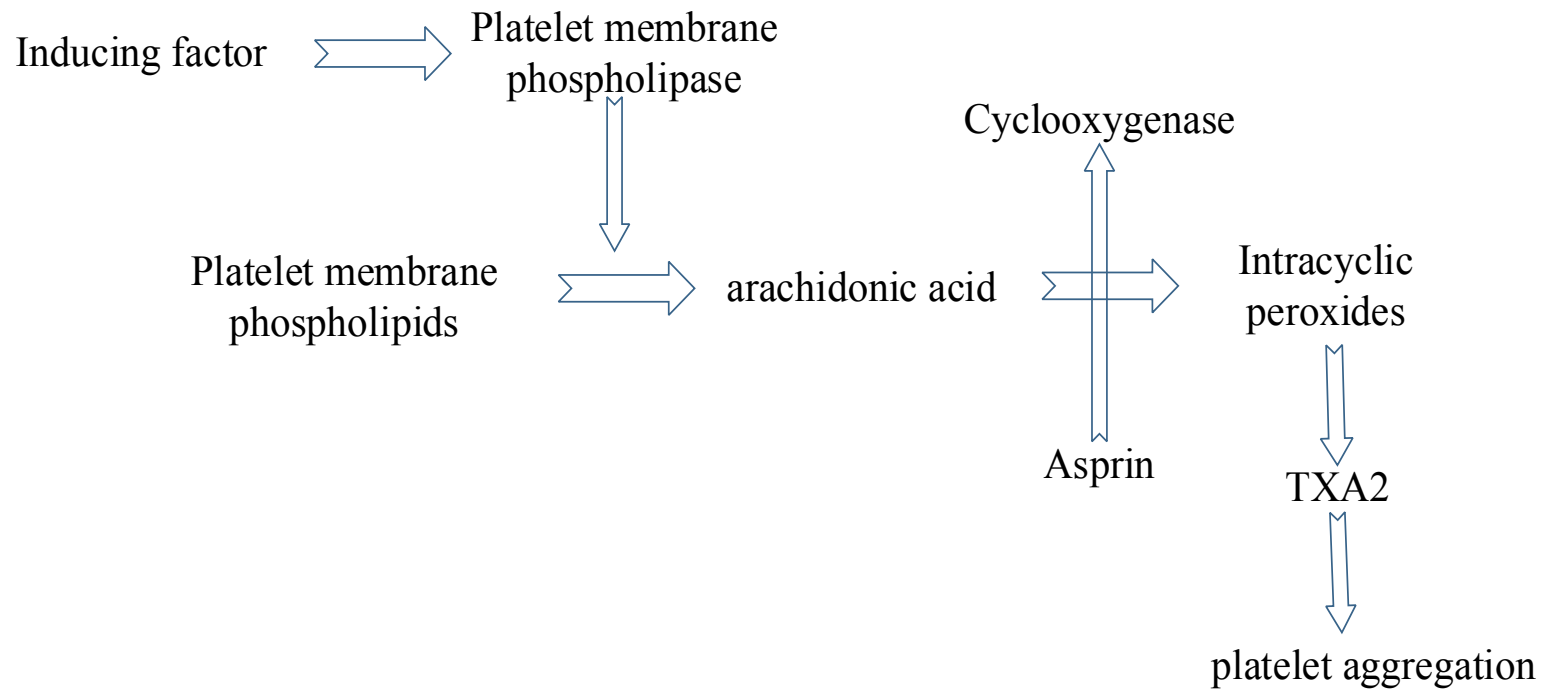

Fig. 1: The generation process of TXA2 in platelet

TABLE 2: COMPARISONS OF ESS RESULTS BETWEEN PATIENTS IN BOTH GROUPS

\begin{tabular}{lcccc}
\hline Group & Before treatment & 1 d after treatment & 7 d after treatment & 14 d after treatment \\
\hline The observation group & $71.57 \pm 13.81$ & $70.88 \pm 17.19$ & $76.04 \pm 19.96$ & $79.51 \pm 17.66$ \\
The control group & $71.08 \pm 13.16$ & $70.95 \pm 17.59$ & $72.43 \pm 16.75$ & $73.21 \pm 15.23$ \\
t & 0.983 & 0.763 & 2.237 & 2.798 \\
P value & 0.112 & 0.174 & 0.027 & 0.015 \\
\hline
\end{tabular}

TABLE 3: COMPARISON OF CLINICAL EFFICACY BETWEEN PATIENTS IN BOTH GROUPS

\begin{tabular}{lcccccc}
\hline Group & Number & Clinical cure & Markedly effective & Effective & Invalid & Total effective rate \\
\hline The observation group & 34 & 4 & 20 & 8 & 2 & $94.12 \%$ \\
The control group & 34 & 1 & 12 & 11 & 10 & $70.59 \%$ \\
$X^{2}$ & - & - & - & - & - & 6.023 \\
$P$ value & - & - & - & - & - & 0.029 \\
\hline
\end{tabular}

TABLE 4: COMPARISON OF NIHSS RESULTS BETWEEN PATIENTS IN BOTH GROUPS

\begin{tabular}{lcccc}
\hline Group & Number & Before treatment & After treatment & P value \\
\hline The observation group & 34 & $4.28 \pm 2.59$ & $3.23 \pm 1.960$ & $<0.05$ \\
The control group & 34 & $4.33 \pm 2.30$ & $3.74 \pm 1.88$ & $<0.05$ \\
$\mathrm{t}$ & - & 0.753 & 2.764 & - \\
$\mathrm{P}$ Value & - & 0.102 & 0.033 & - \\
\hline
\end{tabular}


group. The comparisons and variation tendencies of NIHSS results of patients in both groups were shown in Table 4.

During the course of treatments, 1 case of erythra was found in the observation group and 1 case of nausea and emesis was found in the control group. No abnormalities in the blood and the renal function of patients in both groups were found; the treatments were unaffected after medication adjustments. The differences of side effects between the observation group and the control group were not statistically significant.

Due to its high incidence, high mortality and high disability rate, cerebral infarction has become one of the three major causes of human death along with heart diseases and malignant tumors; in addition, cerebral infarction has become the major cause of death and disability in China. The past epidemiology data indicated that males over $55 \mathrm{y}$ are prone to ischemic cerebral stroke; in addition, based on the statistics, as the age increases every $10 \mathrm{y}$, the incidence rate of stroke approximately doubles. Once the ischemia happens, the perfusion values of cerebral tissues begin to drop, the ischemic tissue neurons could only survive for a couple of hours. The vast majority of ischemic symptoms would develop to the highest peak in seconds or minutes. A few patients would suffer from step-wise or progressive deterioration within a few days and about half of the patients have consciousness disorders while being attacked. Due to the reserve capacity of cerebral perfusion and the compensatory ability of the collateral circulation, if the blood supply is recovered in time, necrosis due to the continued deterioration of ischemia can be avoided, thereby maintaining cerebral functions.

Low molecular weight heparin is produced by chemical or enzymatic depolymerization of HP with a molecular weight of 4500 Dalton; it has high antiXa activity and low antithrombin activity and promotes fibrinolysis. In addition, low molecular weight heparin sodium can enhance the antithrombotic effects of vascular endothelial cells mainly by inhibiting the activity of various blood coagulation factors, interacting with the blood vessel walls and binding low molecular weight heparin to tunica intima to promote the release of endogenous amino polysaccharide. Low molecular weight heparin can change the blood viscosity to enhance the functions of antiaggregation and antithrombosis. In the treatments of cerebral infarction, the anticoagulant system is mainly involved, which prevents thrombus extension and the consequent aggravation of cerebral infarction and the formation of DVT, thereby controlling the complications of cerebral infarction, increasing the neurological function scales of patients, improving the prognosis and preventing recurrence.

The results obtained have suggested that based on the routine aspirin antiplatelet treatments, patients in the observation group was additionally treated with enoxaparin sodium injection and its clinical efficacy was obviously better than that of the control group $(p<0.05)$; in addition, the ESS and NIHSS results of patients in the observation group were better than that in the control group and the differences were statistically significant $(\mathrm{p}<0.05)$; meanwhile, the occurrences of side effects of patients in both groups were not statistically significant ( $p>0.05)$. In summary, the therapeutic effect of enoxaparin sodium injection in combination with aspirin was significant and such treatment plans could effectively improve the neurological deficiency without increasing the risks of hemorrhage, which was worthy of wider clinical applicability. However, the deficiencies of the research process, such as a small sample size and incomplete observation indicators, required further improvement in subsequent studies.

\section{REFERENCES}

1. Jia XF, Hong Z, Fan JH, Zhang YM. Clinical effect of mechanical fragmentation combined with recombinant tissue plasminogen activator artery thrombolysis on acute cerebral infarction. J Biol Regul Homeost Agents 2016;30(3):821-6.

2. Smythe MA, Priziola J, Dobesh PP, Wirth D, Cuker A, Wittkowsky AK. Guidance for the practical management of the heparin anticoagulants in the treatment of venous thromboembolism. J Thromb Thrombolys 2016;41(1):165-86.

3. Sacha GL, Greenlee KM, Ketz JM. The use of anti-factor Xa monitoring in a selection of patients receiving enoxaparin at a large academic medical center. $\mathrm{J}$ Thromb Thrombolys 2016;42(4):1-7.

4. Cimminiello C, Prandoni P, Agnelli G, Di Minno G, Polo Friz $\mathrm{H}$, Scaglione $\mathrm{F}$, et al. Thromboprophylaxis with enoxaparin and direct oral anticoagulants in major orthopedic surgery and acutely ill medical patients: a meta-analysis. Intern Emerg Med 2017;12(2 Suppl):1-15.

5. Korathanakhun P, Petpichetchian C, Petpichetchian W, Sathirapanya P. Comparison of the efficacy of fixed-dose enoxaparin and adjusted-dose unfractionated heparin in patients with cerebral venous thrombosis. Clin Neurol Neurosurg 2017;159:50-4.

6. Martínez-González J, Monreal M, Ayani Almagia I, LlaudóGarín J, Ochoa-Díaz de Monasterioguren L, Gutierro-Adúriz I. Bioequivalence of a biosimilar enoxaparin sodium to Clexane ${ }^{\circledR}$ after single $100 \mathrm{mg}$ subcutaneous dose: results of a randomized, double-blind, crossover study in healthy volunteers. Drug Des Devel Ther 2018;12:575-82.

7. Pouzot-Nevoret C, Barthélemy A, Cluzel M, Verwaerde P, Bonnet-Garin JM, Goy-Thollot I. Enoxaparin has no significant anticoagulation activity in healthy Beagles at a dose of $0.8 \mathrm{mg} /$ $\mathrm{kg}$ four times daily. Vet J 2016;210:98-100. 
8. Roueli A, Cesario E, Amsellem J, Agman A, Vauthier-Brouzes D, Nizard J. Is a therapeutic anticoagulation window needed for delivery when using prophylactic low molecular weight heparin during pregnancy? A retrospective monocentric study. Eur J Obstet Gynecol Reprod Biol 2017;215:118.

9. Dekker KR, Myers BL, Barras MA. Compliance With Enoxaparin Dosing and Monitoring Guidelines and the Impact on Patient Length of Stay: A Pilot Study. Ther Drug Monit 2016;8(1):59-63.
This is an open access article distributed under the terms of the Creative Commons Attribution-NonCommercial-ShareAlike 3.0 License, which allows others to remix, tweak, and build upon the work non-commercially, as long as the author is credited and the new creations are licensed under the identical terms

This article was originally published in a special issue: Special issue on "Drug Development and Human Health in China"

Indian J Pharm Sci 2020:82(1)spl issue2;39-44 